DOI 10.1007/s00167-004-0581-x

\author{
Raffaele Garofalo \\ Cyril Kombot \\ Olivier Borens \\ Ali Djahangiri \\ Elyazid Mouhsine
}

\section{Locking knee caused by subluxation of the posterior horn of the lateral meniscus}

Received: 20 February 2004

Accepted: 17 August 2004

Published online: 11 January 2005

(C) Springer-Verlag 2005

R. Garofalo - C. Kombot - O. Borens

A. Djahangiri · E. Mouhsine $(\bowtie)$

Department of Orthopaedic Surgery and

Traumatology, University Hospital,

OTR-BH 14, CHUV, 1011 Lausanne,

Switzerland

E-mail: Elyazid.Mouhsine@hospvd.ch

Tel: + 41-21-3142791

Fax: + 41-21-3142800

\begin{abstract}
The authors report a case of repetitive locking knee caused by a subluxation of the posterior horn posterior horn was sutured to the posterior knee capsule and the athlete resumed complete sports activity 4 months after the surgery.
\end{abstract} Keywords Lateral meniscus · Knee $\cdot$ Locking $\cdot$ Subluxation

\section{Introduction}

Subluxation or dislocation of an intact lateral meniscus is a very rare and controversial cause of pain and locking knee $[2,4]$.

In literature, the diagnosis and treatment [3], clinical significance and natural history [7] of symptomatic dislocation of anterior horn of medial meniscus, is well described.

On the other hand, to date, we have found only three papers discussing lateral meniscal subluxation [2, 5, 9], and two of these $[5,9]$ report on arthroscopic findings.

We report a case of repetitive knee locking syndrome in a young soccer player, caused by a subluxating posterior horn of a normal lateral meniscus.

\section{Case report}

A 19-year-old footballer experienced his fifth episode of right knee pain and locking, during a stretching session before practice.

He reported that during the preceding four times that the problem had occurred, over a period of 2 year, he had felt excruciating pain and a locking episode of his knee followed by an early and spontaneous resolution. Every time, the episode occurred during sporting activity in an almost full flexion of the knee. No history of trauma was present.

During the most recent, fifth episode, the knee remained locked at $90^{\circ}$ of flexion and a spontaneous unlocking was impossible, leading to the admission of the patient to our institution.

An accurate knee examination was difficult because of pain. After $2 \mathrm{~h}$ from onset of symptoms, the knee presented an extension lag of $30^{\circ}$. Anterior-posterior and lateral radiographs were judged normal. Skin traction was applied over night. The following day the knee was unlocked, less painful and the sports medicine team examined the patient. Clinical examination was within normal limits: no swelling of the knee, no signs of instability were present and $\mathrm{Mc}$ Murray's and Apley's compression/distraction test were negative. During the manoeuvre of knee hyperflexion the patient demonstrated an "apprehension test", he felt an imminent sensation of locking knee. After discussion with the patient a knee arthroscopy was carried out. 


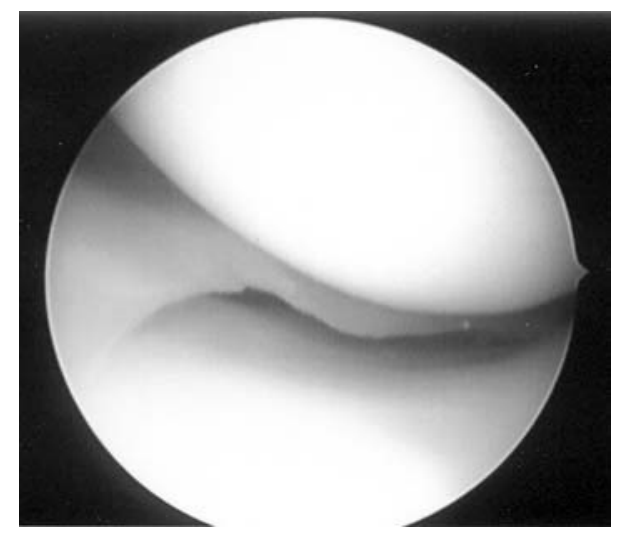

Fig. 1 Normal size and shape appearance of the lateral meniscus

During arthroscopy, we noted a normal femoropatellar joint, no presence of a plica, a normal medial meniscus and cruciate ligaments. The lateral meniscus appeared to be normal in size and shape (Fig. 1). During flexion however, we noted a hypermobility of the lateral meniscus with the posterior horn displacing anteriorly. This hypermobility was better proven using a hook probe (Fig. 2). At this point, the posterior capsule was debrided and an inside-out arthroscopic suture, stabilising the posterior horn of the lateral meniscus to the postero-lateral knee capsule was performed. A prolene $2 / 0$ suture was used, which was passed in a figure of eight pattern as described by Jordan [6]. No further fixation of meniscus to the popliteus tendon was performed to reinforce the repair, because after the suture was performed, the stability of the meniscus was restored.

Post-operatively the knee was immobilised for 3 weeks with a brace in extension. Weight bearing as tolerated was allowed. After 3 weeks, the brace was removed three times each day and an active assisted mobilisation of the knee was started. At 4 weeks, the

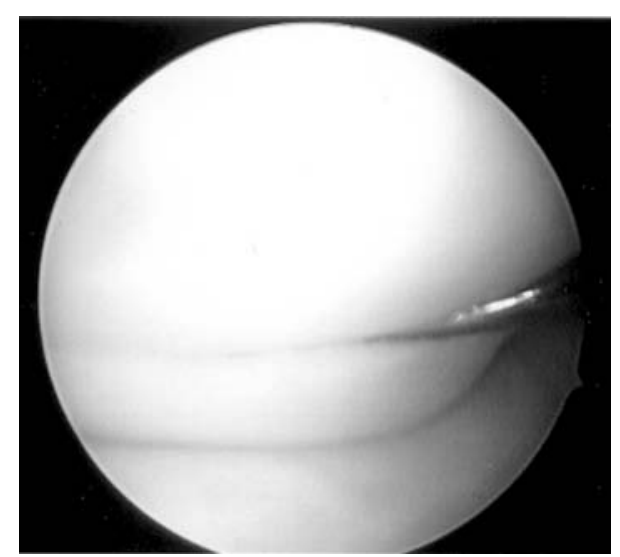

Fig. 2 Hypermobility with subluxation of the lateral meniscus during forced flexion immobilisation was completely discontinued. At 6 weeks, the patient presented an active range of knee motion of 140/0/0 and no imminent sensation of locking was found at clinical examination. At 3 months, lowimpact sports activities were allowed and complete return to sport was possible at 4 months.

At 1 year of follow-up, the patient was satisfied of his level of sports activities and neither complaints nor other episodes of locking knee were experienced.

\section{Discussion}

In contrast to a locked knee caused by the anterior horn of the medial meniscus, which seems to be a well-discussed topic in the literature $[1,3,8]$, the locked painful knee caused by a hypermobile lateral meniscus is rarely reported $[2,5,9]$.

Moreover, it seems that the dislocating horn of the medial meniscus is a normal anatomic variant with little clinical significance and that the need for surgical management is questionable [8]. In the case of an hypermobile, subluxating posterior horn of the lateral meniscus, surgical fixation is indicated as reported by some authors [5, 9] and confirmed by our case. Differing from a precedent report [5], and similar to another [9], there was no true inciting trauma before episodes of locking occurred in our case. Our patient, in fact, complained of pain and locking of the knee after hyperflexion (i.e. stretching of extensor muscles of the thigh). During arthroscopic evaluation no other anomalies of the knee were observed, which differs from an MRI study [2], where a subluxating meniscus was always associated with other internal derangement of knee.

From an anatomic point of view the posterior horn of the lateral meniscus attaches to the tibia behind the intercondylar eminence. The Wrisberg-type discoid meniscus [10] presents a deficient attachment of the meniscotibial ligaments, having only the menisco-femoral ligament of Wrisberg as posterior stabiliser, leading to a potential unstable meniscus and therefore the cause of knee locking.. To date there is no clear explication for a locked knee resulting from a non-discoid variant of lateral meniscus, as in our case and other rare cases [2, 5]. The posterior horn of the lateral meniscus might be susceptible to subluxation, because it has only a loose attachment to the capsule, which is interrupted by the popliteus tendon [7]. Cohn [4] described the anatomy of the popliteus hiatus, showing that the superior border is represented by the posterior superior popliteomeniscle fascicle and the inferior border by the anterior inferior popliteomeniscle fascicle. The occurrence and consistency of fascicular attachments between the lateral meniscus and popliteus tendon are still debated [9]. Simonian et al. [9] reported three cases of lateral meniscus subluxation and they identified disruption of the fas- 
cicular attachments between the popliteus tendon and lateral meniscus as the cause of meniscus instability. These findings were confirmed by MRI study. In our case an MRI study was not performed, to allow an early management of the lesion. On the other hand, in the study of Simonian [9], the MR studies were at first considered normal, showing the difficulty in revealing certain pathological findings overall, when the images are performed with the meniscus in the reduced position. Moreover, because the three cases represented different clinical scenarios, the author [9] did not make a conclusive statement. So it is possible that other types of lesion can also explain this subluxation.

In fact, it may be that the subluxating posterior horn represents a further congenital variant or an anomaly of the lateral meniscus. In certain subjects, the lateral meniscus, although not discoid, can be present with loose menisco-tibial ligaments, so that minimal trauma can result in complete failure of the ligaments and lead to subluxation. All these hypotheses should be confirmed by prospective studies, which may be difficult to perform, because the rarity of this lesion. Moreover, it will not bring many advantages to the treatment, because all the reported cases are well on repairing the posterior horn of the lateral meniscus to the posterior capsule.

About the diagnosis of this lesion, we think as reported by Simonian [9] that an important role is played by clinical history and clinical examination. The MRI study can be useful to eliminate other diagnosis, and it should be correlated to the clinical presentation; otherwise, an overdiagnosis of this injury can occur. Eventually, it should be performed in a locked position of the knee [5] to obtain more information.

Another important point is the resumption of sports activity. On the basis of clinical examination and supposing that the time for a biological scar formation between the posterior horn of the meniscus and the capsule is at least 3 months, we allowed a complete resumption of sports activities at 4 months after surgery. Our result at follow-up was good and showed no sign of recurring subluxation.It was not possible to compare this data, as the relevant literature was lacking.

In conclusion, although not much reported, subluxation of the posterior horn of an intact lateral meniscus can be the only cause of a locking knee.

\section{References}

1. Boucher HH (1964) Anterior marginal separation of the meniscus of the knee. $\mathbf{J}$ Bone Joint Surg Br 46:539-541

2. Breitenseher MJ, Trattnig S, Dobrocky I et al (1997) MR imaging of meniscal subluxation in the knee. Acta Radiol 38:876-879

3. Clancy WG, Keene JS, Goletz TH (1984) Symptomatic dislocation of the anterior horn of the medial meniscus. Am J Sports Med 12:57-64
4. Cohn AK, Mains DB (1979) Popliteal hiatus of the lateral meniscus. Anatomy and measurement at dissection of 10 specimens. Am J Sports Med 7:221-226

5. George M, Wall EJ (2003) Locked knee caused by meniscal subluxation: magnetic resonance imaging and arthroscopic verification. Arthroscopy 19:885888

6. Jordan MR (2000) Lateral meniscal variants. Operative Tech Orthop 10:234-244

7. Mc Murray TP (1949) The semilunar cartilages. Br J Surg 29:407-414
8. Pinar H, Akseki D, Bozkurt M, Karaoglan O (1998) Dislocating anterior horn of the medial meniscus. Arthroscopy 14:246-249

9. Simonian PT, Sussmann PS, Wickiewicz TL et al (1997) Popliteomeniscal fasciculi and the unstable lateral meniscus: clinical correlation and magnetic resonance diagnosis. Arthroscopy 13:590-596

10. Watanabe M, Takeda S, Ikeuchi H (eds) (1979) Atlas of arthroscopy 3rd edn. Igaku-Shoin, Tokyo, pp 75-130 